\title{
Minimal Accesss (TEP) versus Open Inguinal Hernioplasty: A Pragmatic Randomized Control Trial
}

\author{
Ratna Rani Royㄹ, Pankaj Kumar Saha², Md. Abdulla Yusuf ${ }^{3}$, Haridas Saha ${ }^{4}$, Md. Ibnul Hasan ${ }^{5}$, \\ Krishna Pada Saha ${ }^{6}$, Rajib Dey Sarker?
}

${ }^{1}$ Associate Professor, Department of Anatomy, Dr. Sirajul Islam Medical College, Dhaka, Bangladesh; ${ }^{2}$ Senior Consultant \& Coordinator, General and Laparoscopic Surgery, Evercare Hospital, Dhaka \& Former Head \& Professor of Surgery, Shaheed Suhrawardy Medical

College, Dhaka, Bangladesh; ${ }^{3}$ Associate Professor, Department of Microbiology, National Institute of Neurosciences \& Hospital, Dhaka, Bangladesh; ${ }^{4}$ Associate Professor and Head of Surgical Gastroenterology, Sheikh Russel National Gastroliver Institute \& Hospital, Dhaka, Bangladesh; ${ }^{5}$ Assistant Professor, Department of Surgery, Sir Salimullah medical College, Dhaka, Bangladesh; ${ }^{6}$ Resident, Department of Colorectal Surgery, Bangabandhu Sheikh Mujib Medical University, Dhaka, Bangladesh; ${ }^{7}$ Assistant Registrar, Department of Surgery, Shaheed Suhrawardy Medical College Hospital, Dhaka, Bangladesh

[Received on: 22 November 2020; Accepted on: 12 December 2020; Published: 1 January 2021]

\begin{abstract}
Background: Inguinal hernia repair is the most frequently performed operation in general surgery. The standard method for inguinal hernia repair had changed little over a hundred years until the introduction of synthetic mesh. This mesh can be placed by either using an open approach or by using a minimal access laparoscopic technique. Objectives: The purpose of the present study was to compare minimal access laparoscopic mesh techniques with open techniques in hernioplasty. Methodology: This pragmatic randomized control trial was conducted in the Department of Surgery at Shaheed Suhrawardy Medical College \& Hospital, Dhaka from January 2014 to December 2015 for a period of two years. Patients at any age with both sexes who were presented with inguinal hernia were selected as study population. These patients were divided into two group designated as group A and group B. In group A inguinal hernia repair was performed by laparoscopic technique and in group B open technique was used to repair the inguinal hernia. The comparison was done between open and laparoscopic technique of inguinal hernia repair in terms of duration of operation, per-operative complication, immediate post-operative pain, numbness, duration of hospital stay and time of return to normal activities. Follow up was done from 6 months to 2 years. Results: A total number of 200 patients were recruited for repairing of inguinal hernia. Duration of operation was longer initially in the laparoscopic groups (Laparoscopic approach $90 \mathrm{~min}$ vs. Lichtenstein approach $60 \mathrm{~min}$ ). Post-operative pain was another important outcome to consider when choosing between laparoscopic and open repair of inguinal hernia. Laparoscopic repair had been associated with less post-operative pain than open repair. Operative complications were uncommon for both methods. Length of hospital stay was little shorter in laparoscopic group (laparoscopic 1-2 days vs. open technique 3-4 days); however, return to usual activity was earlier for laparoscopic groups (7 days) where open group: 20-30 days. The data available showed less persisting pain (Overall 8/80 versus 12/100) and less persisting numbness (Overall 3/80 versus 7/100) in the laparoscopic groups. Conclusions: In conclusion, minimal access laparoscopic mesh technique is better than open techniques in inguinal hernia repair. [Journal of National Institute of Neurosciences Bangladesh, January 2021;7(1): 75-78]
\end{abstract}

Keywords: Minimal access; TEP; inguinal hernia; hernioplasty; mesh technique

Correspondence: Dr. Pankaj Kumar Saha, Senior Consultant \& Coordinator, General and Laparoscopic Surgery, Evercare Hospital, Dhaka, Bangladesh; Email: pksaha22@gmail.com; Cell no.:+8801711153692

Conflict of interest: All authors had declared that there was no financial conflict of interest of this research work.

Funding agency: Any of the author did not receive any external funding for this study.

Contribution to authors: Roy RR, Saha PK was involved in the concept, study design and data collection. Yusuf MA had prepared the manuscript. Saha H, Hasan MI, Saha KP, Sarker RD have involved in surgery. The Roy RR, Saha PK were involved in proof editing the manuscript.

How to cite this article: Roy RR, Saha PK, Yusuf MA, Saha H, Hasan MI, Saha KP, Sarker RD. Minimal Access versus Open Inguinal Hernioplasty: A Pragmatic Randomized Control Trial. J Natl Inst Neurosci Bangladesh, 2021;7(1):75-78

Copyright: C2021. Roy et al. Published by Journal of National Institute of Neurosciences Bangladesh. This article is published under the Creative Commons CC BY-NC License (https://creativecommons.org/licenses/by-nc/4.0/). This license permits use, distribution and reproduction in any medium, provided the original work is properly cited, and is not used for commercial purposes. 


\section{Introduction}

Inguinal hernia repair is the most frequently performed operation in general surgery ${ }^{1}$. The standard method for inguinal hernia repair had changed little over a hundred years until the introduction of synthetic mesh. This mesh can be placed by either using an open approach or by using a minimal access laparoscopic technique ${ }^{2}$.

Several predisposing factors are reported in cases of inguinal hernia ${ }^{3}$. One of the most common predisposing factor is chronic cough; however, there are some others causes such as from smoking, increases risk of inguinal hernia ${ }^{4}$. In addition there are certain medical conditions which are responsible for the inguinal hernia. Patients who have cystic fibrosis, a life-threatening condition that causes severe lung damage and often a chronic cough, are more likely to develop an inguinal hernia ${ }^{5}$. During chronic constipation straining during bowel movements is a common cause of inguinal hernias. Being moderately to severely overweight puts extra pressure on the abdomen leading to inguinal hernia. Pregnancy can weaken the abdominal muscles and cause increased pressure inside your abdomen ${ }^{6}$.

The laparoscopic revolution in general surgery stimulated by laparoscopic cholecystectomy has also involved the field of inguinal hernioplasty ${ }^{7}$. There is no apparent difference in recurrence between laparoscopic and open mesh methods of hernia repair. The use of mesh during laparoscopic hernia repair is associated with a relative reduction in the risk of hernia recurrence ${ }^{8}$. The data suggests less persisting pain and numbness and reduced post-operative pain following laparoscopic repair. Return to usual activities is faster in laparoscopy. However, operation times are longer and there appears to be a higher risk of serious complication rate in respect of visceral (especially bladder) and vascular injuries". This present study was undertaken to compare the outcome of laparoscopic mesh techniques with open techniques.

\section{Methodology}

This pragmatic randomized control trial was conducted in the Department of Surgery at Shaheed Suhrawardy Medical College \& Hospital, Dhaka, Bangladesh from January 2014 to December 2015 for a period of two years. Patients at any age with both sexes who were presented with inguinal hernia were selected as study population. These patients were divided into two group designated as group A and group B. In group A inguinal hernia repair was performed by laparoscopic technique and total extra-peritoneal approach under general anesthesia were performed among these group. In group $\mathrm{B}$ open approach was used to repair the inguinal hernia and Lichtenstein approach was performed under spinal anesthesia. The patients who were presented with COPD, hypertension, diabetes mellitus or abnormalities of thyroid function were excluded from this study. The comparison was done between open and laparoscopic technique of inguinal hernia repair in terms of duration of operation, per-operative complication, immediate post-operative pain, numbness, duration of hospital stay and time of return to normal activities. Same antibiotic in same doses were applied in both group of patients. The size of mesh in TEP was $15 \mathrm{X} 15 \mathrm{~cm}$. The mesh was fixed by non-absorbable in laparoscopic approach. Follow up was done from 6 months to 2 years. Analyses was performed with SPSS software, versions 22.0 (IBM SPSS Statistics for Windows, Version 22.0. Armonk, NY: IBM Corp.). Continuous data that were normally distributed were summarized in terms of the mean, standard deviation, median, minimum, maximum and number of observations. Skewed data were presented in terms of the maximum, upper quartile, median, lower quartile, minimum and number of observations. Categorical or discrete data were summarized in terms of frequency counts and percentages. Baseline characteristics were presented by treatment group. When values were missing, the denominator were stated. Continuous variables were summarized as a mean (with SD).

\section{Results}

A total number of 200 patients were recruited for repairing of inguinal hernia. Duration of operation was longer initially in the laparoscopic groups (Laparoscopic 90 min vs. Open $60 \mathrm{~min}$ ). Post-operative pain was another important outcome to consider when choosing between laparoscopic and open repair of inguinal hernia. Laparoscopic repair had been associated with less post-operative pain than open repair.

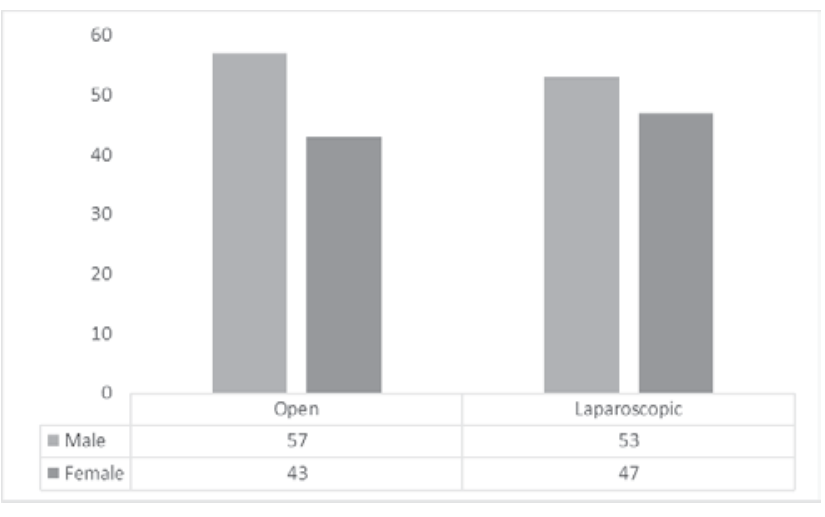

Figure I: Gender Distribution among Study Population 
Table 1: Comparison of Open and Laparoscopic Technique during Surgery

\begin{tabular}{|c|c|c|}
\hline Parameter & Open technique & Laparoscopic technique \\
\hline Total & 100 & 100 \\
\hline Duration of operation & 60 minutes(Average) & $\begin{array}{c}\text { Initially } 90 \text { min but later on same as } \\
\text { open technique }\end{array}$ \\
\hline Per-operative complication & Nil & Nil \\
\hline Immediate Post-operative pain & $\begin{array}{l}\text { More post-operative pain so, } \\
\text { more analgesic requirement }\end{array}$ & $\begin{array}{l}\text { Less post-operative pain so, } \\
\text { less analgesic requirement }\end{array}$ \\
\hline Duration of hospital stay & 3 to 4 days (little more) & 1 to 2 days (little shorter) \\
\hline Return to normal activities & 3 to 4 weeks & Earlier (average 7 days) \\
\hline
\end{tabular}

Per-operative complication=visceral injuries, vascular injuries

Operative complications were uncommon for both methods. Length of hospital stay was little shorter in laparoscopic group (laparoscopic 1 to 2 days vs. open technique 3 to 4 days); however, return to usual activity was earlier for laparoscopic groups (7 days) where open group: 20 to 30 days. The data available showed less persisting pain (Overall 8/80 versus 12/100) and less persisting numbness (Overall 3/80 versus 7/100) in the laparoscopic groups. There was no apparent difference in recurrence between laparoscopic and open mesh methods of hernia repair. The use of mesh during laparoscopic hernia repair was associated with a relative reduction in the risk of hernia recurrence. The data suggested less persisting pain and numbness and was reduced post-operative pain following laparoscopic repair. Return to usual activities was faster in laparoscopy. However, operation times were longer and there appears to be a higher risk of serious complication rate in respect of visceral especially bladder and vascular injuries (Table 1).

Considering the post-operative outcomes chronic pain and numbness were reported more in open surgery than laparoscopic surgery. In open surgery 10 patients had chronic pain and 8 patients had numbness; however, in laparoscopic surgery group 5 patients had reported chronic pain and 2 patients had presented with numbness. Recurrence was reported in 1 patient in open surgery; on the other hand laparoscopic surgery group 2 patients were developed recurrence. Post-operative

Table 2: Post-Operative Outcomes after Open and Laparoscopic Surgery

\begin{tabular}{lcc}
\hline Post-Operative & \multicolumn{2}{c}{ Tye of Infection } \\
\cline { 2 - 3 } Outcomes & Open & Laparoscopic \\
\hline Chronic Pain & 10 & 5 \\
Numbness & 8 & 2 \\
Recurrence & 1 & 2 \\
Post-operative complication & 4 & 1 \\
\hline
\end{tabular}

Post-operative complication=seroma, wound infection completions were reported in 4 patients of which 3 patients developed seroma and 1 patient had wound infection. In laparoscopic group, only 1 patient developed seroma but no wound infection or mesh infection was reported (Table 2).

\section{Discussion}

There are several techniques of inguinal hernia repair that includes open repair, open tension free repair and endoscopic repair with mesh. More than 100 years ago Edoard Bassini introduced his technique to repair groin hernia, since then variety of technique developed for open repair of inguinal hernia. Despite all effort the recurrence rate has been the same ranging from $5 \%$ to $10 \%$ and average time to resume normal activities remain 21 to 30 days $^{10}$. In 1984 Lichtenstein used mesh for tension free reinforcement of inguinal floor with reduced recurrence rate. The laparoscopic revolution in general surgery stimulated by laparoscopic cholecystectomy has also involved the field of inguinal hernioplasty ${ }^{11}$.

Laparoscopy is beneficial to the surgeons which includes safe, better visualization, improved dissection with reduced blood loss using harmonic scalpel, potential benefits for training, potential to improve quality, reduced post-operative pain, reduced hospital stay and improve cosmesis ${ }^{12}$. Again, the laparoscopy is also beneficial to the patients which includes less scarring, less pain, shorter hospital stay and quicker return to activities.

Per-Operative Complication: Although per-operative complication like visceral injuries, vascular injuries are more common in laparoscopic technique but in this study there is no complication. Similar findings are also reported in other studies ${ }^{11-14}$.

Post-operative Pain: Post-operative pain is important outcome to consider when choosing between laparoscopic and open repair of inguinal hernias. Laparoscopic repair has been associated with less post-operative pain than open repair ${ }^{15}$. 
Chronic Pain: When making the decision for open or laparoscopic inguinal hernia repair, differentiating between the chronic pain associated with each method is another important consideration less persisting pain in the laparoscopic groups ${ }^{16}$.

Return to work: Another variable that is used as a primary outcome in numerous studies comparing laparoscopic and open techniques is return to work. The patients who undergo laparoscopic inguinal hernia repair return to work and normal activities more rapidly than those who undergo open repair ${ }^{12}$. A quicker return to work and resumption of normal activity is associated with an earlier discharge from the hospital and fewer post-operative complications, both of which are associated with laparoscopic hernia repair ${ }^{15}$.

Operation Length and Technical Difficulty: Considering the goal of improving patient care, it would follow that respective outcomes would drive the decision between open and laparoscopic inguinal hernia repair $^{15}$. Although outcomes may be the primary consideration, it is still important to consider the factors that impact the efficiency of each procedure. With regard to operation length, most evidence in a shorter operation duration are with open repair ${ }^{14}$. The difference in the duration of the operation can be partly attributed to operative complications, which although uncommon for both methods, are more frequent in the laparoscopic group for vascular injuries ${ }^{16}$.

Hernia Recurrences: Recurrence is arguably the most important indicator of the success of a hernia procedure. Hernia recurrences after surgical repair may occur in $15 \%$ of the cases or more ${ }^{11}$. The inguinal hernia repairs suggest no apparent difference in recurrence between laparoscopic and open mesh methods of hernia repair. A separate meta-analysis published in 2000 reported similar findings in that overall recurrences did not differ between the laparoscopic and open groups ${ }^{13}$.

\section{Conclusion}

However, there is no apparent difference in recurrence between laparoscopic and open mesh methods of hernia repair. The use of mesh during laparoscopic hernia repair is associated with a relative reduction in the risk of hernia recurrence. The data suggests less persisting pain and numbness and reduced post-operative pain following laparoscopic repair. Return to usual activities is faster in laparoscopy. However, operation time is longer in TEP, it is likely that surgeon's preference will continue to dictate the approach employed in hernia repair for the foreseeable future.

\section{References}

1. MRC Laparoscopic Groin Hernia Trial Group. Laparoscopic versus open repair of groin hernia: a randomised comparison. The Lancet. 1999;354(9174):185-90.

2. Antoniou SA, Antoniou GA, Bartsch DK, Fendrich V, Koch OO, Pointner R, et al. Transabdominal preperitoneal versus totally extraperitoneal repair of inguinal hernia: a meta-analysis of randomized studies. The American Journal of Surgery. 2013; 206(2):245-52.

3. Darzi A, Mackay S. Recent advances in minimal access surgery. Bmj. 2002;324(7328):31-4.

4. Eker HH, Langeveld HR, Klitsie PJ, van't Riet M, Stassen LP, Weidema WF, et al. Randomized clinical trial of total extraperitoneal inguinal hernioplasty vs Lichtenstein repair: a long-term follow-up study. Archives of Surgery. 2012;147(3): 256-60.

5. Jeans S, Williams GL, Stephenson BM. Migration after open mesh plug inguinal hernioplasty: a review of the literature. The American surgeon. 2007;73(3):207-9.

6. Lau H, Patil NG, Yuen WK. Day-case endoscopic totally extraperitoneal inguinal hernioplasty versus open Lichtenstein hernioplasty for unilateral primary inguinal hernia in males. Surgical Endoscopy and Other Interventional Techniques. 2006;20(1):76-81

7. Fan JK, Yip J, Foo DC, Lo OS, Law WL. Randomized trial comparing self gripping semi re-absorbable mesh (PROGRIP) with polypropylene mesh in open inguinal hernioplasty: the 6 years result. Hernia. 2017;21(1):9-16.

8. Lau H, Patil NG, Yuen WK, Lee F. Prevalence and severity of chronic groin pain after endoscopic totally extraperitoneal inguinal hernioplasty. Surgical Endoscopy and Other Interventional Techniques. 2003;17(10):1620-3.

9. Lau H. Patients' perception of open and endoscopic extraperitoneal inguinal hernioplasty. Surgical Laparoscopy Endoscopy \& Percutaneous Techniques. 2004;14(4):219-21.

10. Gürleyik E, Gürleyik G, Çetinkaya F, Ünalmiser S. The inflammatory response to open tension-free inguinal hernioplasty versus conventional repairs. The American journal of surgery. 1998;175(3):179-82.

11. Palumbo P, Minicucci A, Nasti AG, Simonelli I, Vietri F, Angelici AM. Treatment for persistent chronic neuralgia after inguinal hernioplasty. Hernia. 2007;11(6):527-31.

12. Himpens JM. Laparoscopic inguinal hernioplasty. Surgical endoscopy. 1993;7(4):315-8.

13. Forte A, D'urso A, Palumbo P, Storto GL, Gallinaro LS, Bezzi $\mathrm{M}$, Beltrami V. Inguinal hernioplasty: the gold standard of hernia repair. Hernia. 2003;7(1):35-8.

14. Sözen S, Çetinkünar S, Emir S, Yazar FM. Comparing sutures and human fibrin glue for mesh fixation during open inguinal hernioplasty. Ann Ital Chir. 2012 Jul.

15. Lau H. Endoscopic totally extraperitoneal inguinal hernioplasty for recurrence after open repair. ANZ journal of surgery. 2004;74(10):877-80.

16. Peitsch WK. Laparoscopic transperitoneal inguinal hernioplasty (TAPP) after radical open retropubic prostatectomy: special features and clinical outcomes. Hernia. 2019;23(2):281-6 